\title{
Awake Aortic Surgery in a Tetraparetic Patient
}

\author{
Luca Gramaglia $^{*}, 1$, Piero Brustia ${ }^{1}$ and Umberto Massazza ${ }^{2}$ \\ ${ }^{1}$ University Hospital, Novara, Italy \\ ${ }^{2}$ Biella Hospital, Biella, Italy.
}

\begin{abstract}
A greater number of people with spinal cord injury is always submitted to surgical operations. Some pathologies associated to the medullary damage as the autonomic dysreflexia, the muscular spasm and the respiratory inadequacy can increase perioperative complications. Manifold early studies have shown that the multimodal approach in complex elective surgery can reduce perioperative morbility and mortality.

We describe the case of a 77 year-old patient tetraparetic scheduled for open abdominal aortic aneurismectomy using multimodal approach that consists in minimal invasive surgery (subcostal incision), thoracic epidural anesthesia with light sedation and postoperative forced rehabilitation. This approach has allowed the decrease of perioperatorie complications and the improvement of postoperative outcome.
\end{abstract}

\section{INTRODUCTION}

An increasing number of patients with spinal cord injury requires surgical care. Their most important perioperative problems are: autonomic dysreflexia, bradycardia, hypotension, respiratory inadequacy and muscle spasm [1]. In the abdominal aortic aneurismectomy, a high risk surgery, these problems increase the complications [2]. In the last years there was a growing agreement about the safety of spinal anesthesia in these patients [1]; in fact there are many advantages in epidural anesthesia: reduction of stress response, hemodynamic stability, improvement in postoperative pulmonary and gastrointestinal function and reduction of thromboembolic complication [3]. Latest studies have demonstrated that the multimodal approach in complex elective surgical procedures may lead to a major reduction of postoperative morbidity with improved postoperative outcome. This approach includes: minimal invasive surgery, thoracic epidural anesthesia and enforced postoperative rehabilitation $[4,5]$. We describe a case of tetraplegic patient scheduled for open abdominal aortic surgery, performed with minimal subcostal incision, epidural thoracic anesthesia and a program of fast rehabilitation.

\section{CASE HISTORY}

A 77-year-old men with tetraparesis was scheduled for open abdominal aortic surgery. The aorta presented $5,5 \mathrm{~cm}$ aneurysm, with diameter increased more than $1 \mathrm{~cm}$ in the last 6 months. The anatomical features excluded endovascular procedures. Nine years before the aneurismectomy, the patient, formerly suffering from a cervical spine stenosis and multiple intervertebral cervical disc herniation (from $\mathrm{C} 4$ to C6), suddenly developed a spondylogenic myelopathy owing to a cervical spine trauma. He underwent a laminectomy $\mathrm{C} 4-$ C5-C6 and stabilization with plates two months later. In spite of the surgical decompression, the physical neurological pattern and the electrophysiological findings didn't improve in

*Address correspondence to this author at the University Hospital, Novara, Italy; Fax: 00393213733253; E-mail: lucagramaglia@email.it subsequent years and the patient outcome was a tetraparesis with neurological bladder, requiring daily intermittent catheterism. The strength loss was more pronounced distally both in upper and lower limbs and the standing position and the walking were lost. As regard to the sensibility deficit, the patient shows a level of hypoesthesia from bisacromial line distally, together with scattered skin areas of hypersensitiveness below the spinal lesion. Finally, we found muscle spasm, provoked by stimuli even negligible and controlled with lorazepam 3 $\mathrm{mg}$ /day. At the admission time the patient had: neurological bladder and extemporaneous catheter; moderate chronic renal failure (serum creatinine: $1.2 \mathrm{mg} / \mathrm{dl}$, creatinine clearance: 40ml/min, urinary osmolarity: $265 \mathrm{mOspm} / \mathrm{KgHzO}$ ) caused by chronic pielonephrity; chronic constipation; chronic sideropenic anemia with Ferro therapy; hypertension treated with calcium channel blockers. Moreover, the patient assumed daily: $20 \mathrm{mg}$ diazepam, $100 \mathrm{mg}$ allopurinol and $24 \mathrm{mg}$ senna. At the physical examination- the patient was in adequate condition, with body mass index of 21,35; the lungs had MV spread reduced; EGA e PFR were normal; the cervical spine was inflexible; Mallampati was 3 and Patil less than $5 \mathrm{~cm}$. The strategy chosen was: minimal invasive surgery, thoracic epidural anaesthesia and enforced postoperative rehabilitation.

Premedication was with $6 \mathrm{mg}$ morphine intramuscularly two hours before surgery. Following insertion of two large bore venous cannulae in the forearms, $2 \mathrm{~g}$ ceftazidime, 100 $\mathrm{mg}$ ketoprofen and $10 \mathrm{mg}$ metoclopramide iv were administered. After insertion of catheter in the radial artery to monitor invasive arterial blood pressure, $100 \mathrm{mcg}$ fentanyl, 2,5 $\mathrm{mg}$ droperidol and $1 \mathrm{mg}$ midazolam iv were administered. Patient received $3 \mathrm{l} / \mathrm{min} \mathrm{O}_{2}$ by facial mask. Epidural catheter was placed at T7-T8 interspace and $25 \mathrm{ml}$ bupivacaine 0.5 per cent administered in the space of 1 hour, achieving sensory block (pin prick on sensible zones) between T4 and L5 dermatomes after 40 minutes. Then urinary drainage was inserted. Epidural infusion of $0,5 \%$ bupivacaine at a rate of $4-5 \mathrm{ml} / \mathrm{h}$ was maintained throughout 
surgery. A total of $1100 \mathrm{ml}$ of fluids was administered: 500 $\mathrm{ml}$ of $10 \%$ hydroxyethyl starch and $600 \mathrm{ml}$ of crystalloids. A thermal blanket in contact with the exposed parts of the upper body was used to maintain normothermic the patient. The aortic by pass was performed with transperitoneal access. With the patient in supine position, a curvilinear left subcostal incision, parallel to the costal edge, of $13 \mathrm{~cm}$ long was made. The viscera were left in the abdominal cavity and manually displaced on the right side without the use of selfretaining retractors. Once the aortic graft was inserted (time of clamp: 46 minutes), the abdominal wall was closed with no drains. No nasogastric tube was inserted. Surgical time was 75 minutes; blood 10ss was $150 \mathrm{ml}$. Verbal contact with the patient was maintained during surgery. Throughout the operating time, the patient never felt discomfort. No dangerous hemodynamic changes were registered: (Fig. 1). $15 \mathrm{mg}$ ephedrine iv were administered only during induction of epidural anesthesia, to prevent hypotension. The urinary output was $95 \mathrm{ml} / \mathrm{h}$. Peripheral 02 saturation remained more than $98 \%$. Blood gas analysis showed an optimal compensation during the clamping and declamping of the aorta (Fig. 2). At the end of surgery, radial catheter was removed and the patient was transferred to the surgical ward. Analgesia was achieved with a continuous epidural infusion of $0.25 \%$ bupivacaine at a rate of $3-6 \mathrm{ml} / \mathrm{h}$ for 48 hours orally, supplemented by $600 \mathrm{mg}$ ibuprofen every eight hours. Pain control, evaluated with VAS (visual analog scale) was optimal: VAS at rest and VAS on coughing remained 0 during the day of surgery (day0), day 1, day 2 and day 3 . Hemodynamic parameters remained stables: (Fig. 3). During the three days after surgery respiratory rate varied from 19 to $21 \mathrm{breaths} / \mathrm{minutes}$ and peripheral $\mathrm{O}_{2}$ saturation varied from 96 to $99 \%$ (patient received $0_{2}$ supplementation by mask during the first two postoperative nights). $\mathrm{Rx}$ chest ray performed in day 2 was unchanged in comparison with preoperative one. After surgery, once general conditions

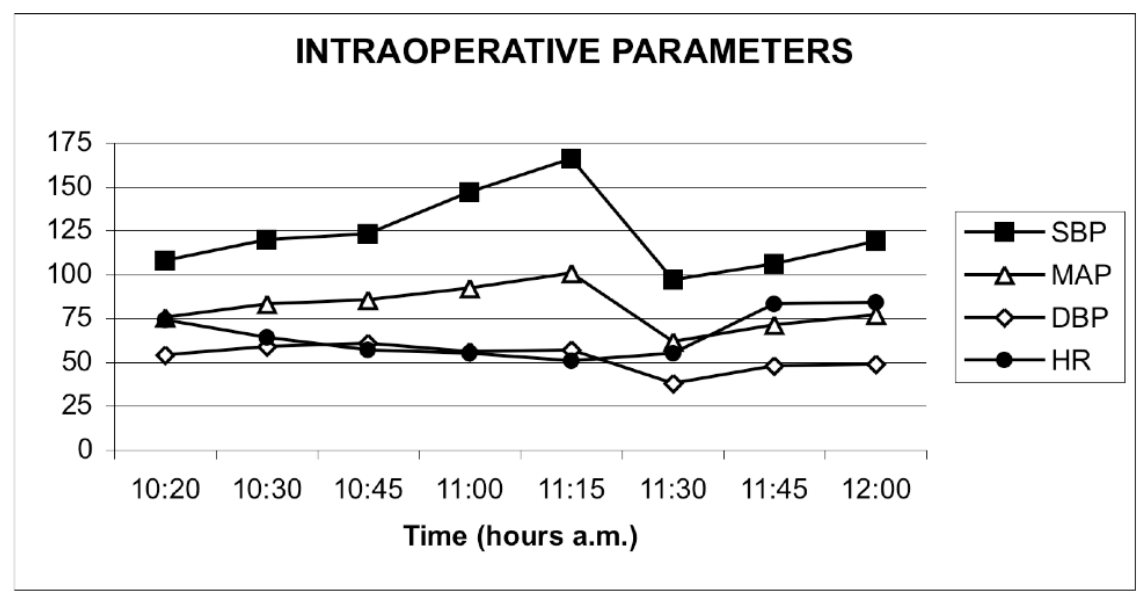

SBP: systolic blood pressure; MAP: mean arterial pressure; DBP: diastolic blood pressure; HR: heart rate Fig. (1).

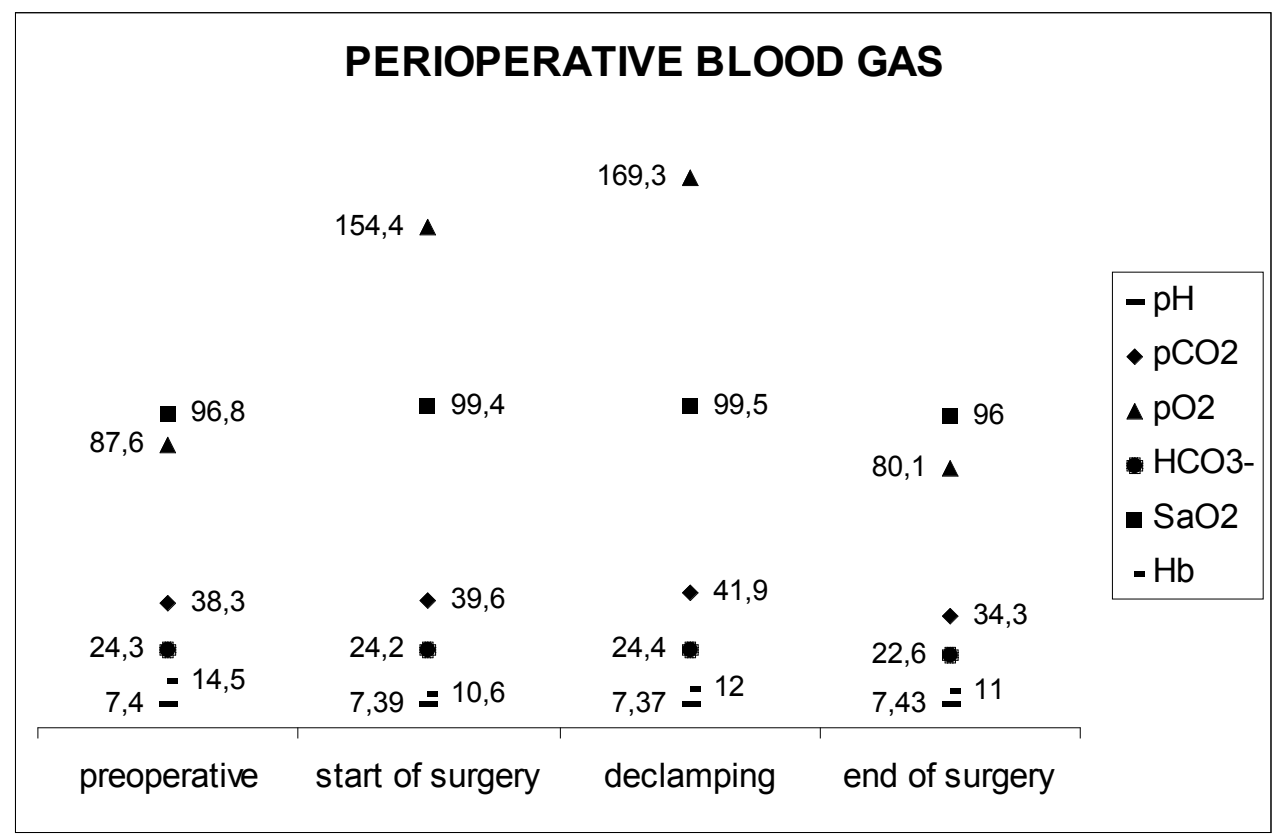

Fig. (2). 


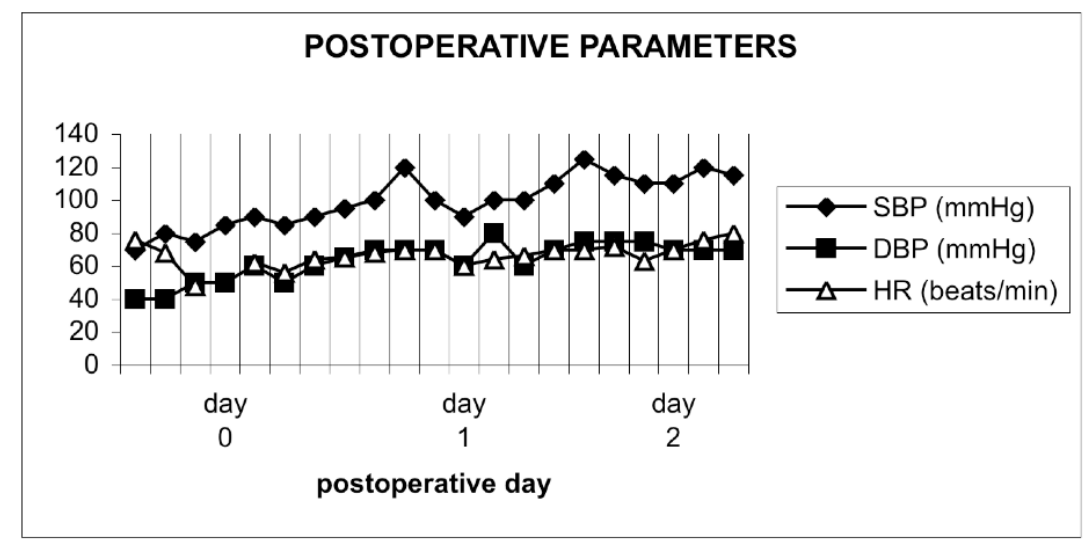

SBP: systolic blood pressure; DBP: diastolic blood pressure; HR: heart rate

Fig. (3).

were stable, patient was stimulated to drink freely sugared beverages. A semisolid diet was offered two hours after surgery. $10 \mathrm{mg}$ metoclopramide was administered orally every 8 hours to improve gastric peristalsis. In day 0 patient intake was $1760 \mathrm{ml}$ of oral fluids, $812,4 \mathrm{Kcal}$ (43\% of Kcal needed) and $14,3 \mathrm{~g}$ of proteins with oral diet. In day 1 patient intake was $2215 \mathrm{ml}$ of oral fluids, $1488,4 \mathrm{Kcal}(78,9 \%$ of Kcal needed) and 54,94 g of proteins with oral diet. Passage of stools was at $42^{\wedge}$ postoperative hour. After surgery, patient was immediately enforced to perform breathing exercises and foot pump with active and passive exercises. Patient was mobilized out of bed with assistance three hours after surgery. In the follow- up of postoperative days patient was mobilized in chair 5 hours/day. Bladder catheter was removed on the third day after surgery and extemporaneous catheter was started. Chemistry showed a transient increase of serum creatinine $(1,6 \mathrm{mg} / \mathrm{dl})$ without clearance changes in day 2 and with return to baseline values in the subsequent days. Patient was discharged at home in fourth postoperative day. A good outcome was confirmed during a consultation with medical team, planned 8 days after operation and the echocolordoppler 6 months later.

\section{DISCUSSION}

In the last years, the improvements in the care of patients with spinal cord injury have lead to decrease morbidity and mortality, so these patients are scheduled for surgery. The pathophysiological changes caused by spinal cord injury increase anesthesiological risk. Autonomic dysreflexia, more frequent with lesions higher then T7, can cause paroxysmal and severe hypertension in response to stimuli below the region of spinal injury. Such hypertensive crises can lead cardiovascular accident. Respiratory impairment results of muscle weakness and paralysis of abdominal wall muscles impairs the ability to cough. Surgical stimulus can provoke violent muscle spasms. Bladder and gastric emptying are delayed; renal failure is common. The perioperative increase if patient is scheduled for aortic surgery, associated with cardiac, respiratory, renal and gastrointestinal complications, resulting from pathophysiological disturbances that occur during cross-clamping and unclamping [2]. Many studies indicate that neuraxial anesthesia is safe and favourable to patients with chronic spinal cord injury $[6,7]$. Particularly, continuous epidural anesthesia-analgesia may control autonomic hyperreflexia and stimulate enteral motion in low abdomen surgery [7]. There are many advantages in epidural anesthesia-analgesia, also in high-risk surgical patients: reduction of stress response, hemodynamic stability, improvement in postoperative pulmonary and gastrointestinal function, reduction of thromboembolic complications $[3,8]$. Nevertheless, there is no evidence that perioperative epidural analgesia significantly influences major morbidity or mortality [9]. Several authors demonstrated but no single technique has been shown to eliminate postoperative complications, yet multimodal intervention in patients scheduled to complex elective surgical procedures may lead to a major reduction of postoperative morbidity with improved postoperative outcome. This approach includes: minimal invasive surgery, thoracic epidural anesthesia and enforced postoperative rehabilitation $[4,5]$. In our Institution we usually apply this approach, that team has decided to use for these case too. Surgical approach consists in minimal subcostal incision [10].

Thanks to thoracic epidural anesthesia, we performed awake aortic surgery repair with good comfort for the patient. Also in postoperative we registered an optimal pain control. In the perioperative time hemodynamic and respiratory parameters remained stable. Immediately after surgery patient was mobilized and received oral diet without any problem. In a day after surgery patient Consumed $78 \%$ of caloric needs. Patient was discharged at home in day 4 after surgery, in general condition as well as preoperative.

To conclude, the multimodal approach, marked by mininvasive surgery, epidural anesthesia-analgesia and enforced postoperative rehabilitation, has shown to be effective to decrease perioperative complications and improve outcome $[11,12]$. The technique, applied in this situation, where there are further problems due to basal pathology (as autonomic dysreflexia), seemed to be equally safe and effective in this particular case.

\section{REFERENCES}

[1] Hambly PR, Martin B. Anesthesia for chronic spinal cord lesion. Anesthesia 1998; 53: 273-89.

[2] Gelman S. The Pathophisiology of aortic cross-clamping and unclamping. Anesthesiology 1995; 82: 1026-60. 
[3] Liu S, Carpenter RL, Neal JM. Epidural anaesthesia and analgesia. Anesthesiology 1995; 82: 1474-506.

[4] Carli F, Klubien K. Thoracic epidurals: is analgesia all we want? Can J Anaesth 1999; 46 (5): 409-14.

[5] Kehlet H. Multimodal approach to control postoperative pathophisiology and rehabilitation. Br J Anesth 1997; 78: 606-17.

[6] Okuyama A, Veda M, Morimoto Y, Okuyama M, Kemmotsu O. Anesthetic management for urological surgery of patients with chronic spinal injury. Masui 1994; 43 (7): 1033-7.

[7] Vigreux G, Vacant J, Lepresle E, Desalle De Hys C, Debras C. A propos de suites opèratoires des tétraplégiques en chirurgie urologique. J Urol Néphrol 1976; 6: 411-6.
[8] Yeager MP, Glass DD, Neff RK, Brinck-Johnsen T. Epidural anesthesia and analgesia in high-risk surgical patients. Anesthesiology 1987; 66: 729-36.

[9] Peyton PJ. Myles PS, Silbert BS, et al. Perioperative epidural analgesia and outcome after major abdominal surgery in high-risk patients. Anesth Analg 2003; 96: 548-54.

[10] Brustia P, Porta C. Left sub costal minilaparotomy in aortic surgery. Minerva Cardioangiol 2001; 49: 91-7.

[11] Rodgers A, WaIker N, Schug S, et al. Reduction of postoperative mortality and morbidity with epidural or spinal anesthesia: results from overview of randomised trials. BMI 2000; 321: 1-12.

[12] Wilmore DW, Kehlet H. Management of patients in fast track surgery. BMJ 2001; 322: 473-6.

(C) Gramaglia et al.; Licensee Bentham Open.

This is an open access article licensed under the terms of the Creative Commons Attribution Non-Commercial License (http://creativecommons.org/licenses/by$\mathrm{nc} / 3.0 /$ ) which permits unrestricted, non-commercial use, distribution and reproduction in any medium, provided the work is properly cited. 